\title{
Application of Moiré Interferometry for Measuring API Gravity of Crude Oil
}

\author{
H.El Ghandoor and E.A.Abo Mostafa \\ Faculty of Science Ain Shams University, tnemtrapeD scisyhP \\ Abbassia 11566, Cairo Egypt
}

\begin{abstract}
In this work two concentric capillary tubes-lense interferometers have been used with the same laser focused sheet of light. One of the lense-capillary tube interferometers was filled with the crude oil under investigation, while the second lense-capillary tube interferometers was filled with air at room temperature and normal pressure. The transverse interference fringes produced from each of the interferometers were allowed to be combined together displaying the Moire interference fringe pattern that can be used for the calculations of the crude oil refractive index and density. Also PC software was performed to extract the information needed from the resulting Moiré pattern to obtain the API gravity of the oil. (American Petroleum Institute)
\end{abstract}

\section{INTRODUCTION}

Optical properties, such as refractive index, absorption coefficient, and radiance are fundamental parameters of crude oils useful not only in remote-sensing detection but also in a number of industrial applications. The refractive index, in particular, is used in petroleum separation processes, such as in predicting the onset of asphaltene precipitation $^{1}$ and in measuring the solubility parameter $^{2}$, and in measurements of oil film thickness. In addition, it plays a vital parameter in various theoretical models related to specific compositions of crude oil and its fractions ${ }^{3}$. However, there has been no formal endorsement for the refractive index to be used as a true fingerprinting tool for crude oils. The main reason for this is the refractive indices of crude oils can not measured by dispersion devices such as Abbe's refract meter due to

the high absorption coefficient of the crude oil. Although there are more accurate methods $^{4-10}$ to determine the refractive index of fluids, but all require complicated setups and thus accordingly, cannot be straightforwardly applied.

In the present letter we describe a new simple Moiré Interferometric method to measure the refractive index of crude oils (or fluids in general), which requires neither an elaborate setup nor a reference sample and can provide accuracy up to $10^{-7}$. With such simple setup and high accuracy $\left(\sim 10^{-7}\right)$ the refractive index of crude oils would be suitable to be considered as bar codes by which crude oils can be practically labeled. The method was recently developed to examine optical fibers ${ }^{11,}{ }^{12}$, and has been modified in this work in such a way that capillary tubes filled with crude oil samples replaced the optical fibers. Because the resulting interference patterns in this method is localized they can also be easily detected in reflection, making this method (and its theoretical model) developable for remote sensing measurements. The method can be considered, as an alternative method for measuring the refractive index of liquids inside capillary tube by using two lens - capillary tube interferometers. 
Military Technical College

Kobry Elkobbah,

Cairo, Egypt

May 29-31,2012

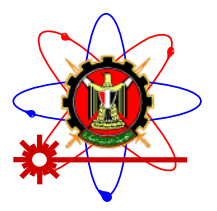

$6^{\text {th }}$ International Conference

on Mathematics and

Engineering Physics

(ICMEP-6)

Instead of using one lens - capillary tube interferometer filled with the liquid under test, two coaxial lens-capillary tube interferometer has been used.

In this case two transverse interference patterns are obtained in the image plane they combine coherently with each other to produce Moiré pattern each corresponds to an optical path difference equal to $(\lambda / 2 \sin \alpha)$ where, $\lambda$ is the wave length of the used laser source, and

$\alpha$ is the angle subtended by each of the two interferometer with the screen. The recorded two transverse interference patterns from each of the two lense-capillary tube interferometer, presents the basis of the method used in this paper. This is to produce a Moiré pattern that can be observed when the recorded two patterns are overlapped and observation takes place along the direction of the direct first order diffraction image. The transverse interference patterns lines run perpendicular to the line joining the centers of the two lense-capillary tube interferometer,

If $\alpha$ is the half - angle between rays emerging from the two lense-capillary tube interferometer, the spacing of the transverse interference fringes is :

$$
\delta=\lambda / 2 \sin \alpha
$$

Assuming the small angle approximation

$$
\tan \alpha=\sin \alpha=\mathrm{D} / 2 . \mathrm{L}
$$

The formed Moiré pattern, which are contours of equal in-plane path difference between the two interferometers, where the spacing of the Moire fringes corresponds to displacement of: $\lambda . \mathrm{L} D$

\section{EXPERIMENTAL SETUP}

A schematic diagram of the experimental setup is shown in Fig. 1. two coaxial Calibrated capillary tubes (inner and outer diameters $1.0 \mathrm{~mm}$ and $1.4 \mathrm{~mm}$, respectively) the inner one was filled with crude oils while the outer was open to air. The two coaxial tubes were placed on a fixed holder, one at a time, and then illuminated by a laser sheet from a 10$\mathrm{mW}$ He-Ne laser in the z-direction. The sheet was formed by passing the laser beam through a microscope-objective lens, a collimating lens, and a cylindrical lens whose axis was parallel to the axis of the capillary tube (along the x-direction). The formed sheet had a length of approximately $3 \mathrm{~cm}$, and a thickness wide enough to illuminate the whole width of the two capillary tubes. The resulting two transverse interference patterns were formed at the two capillary tube-lens interferometers itself and also in the region behind it where it was projected onto a fixed screen whose plane was kept perpendicular to the $\mathrm{z}$-axis. The two transverse interference patterns are obtained in the image plane and then

combined coherently with each other to produce Moiré interference fringe pattern each corresponds to an optical path difference equal to 
Military Technical College

Kobry Elkobbah,

Cairo, Egypt

May 29-31,2012

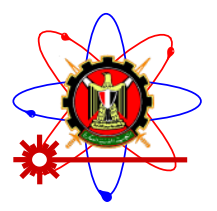

$6^{\text {th }}$ International Conference

on Mathematics and

Engineering Physics

(ICMEP-6)

\section{$\lambda / 2 \sin \alpha$}

where, is the wave length of the used laser source, A digital image of the projected two transverse interference patterns were then recorded using a CCD camera, stored in a computer and later retrieved for carrying out the analysis.

Seven blended petroleum crude oils, named as A, B, C, D, E, F, and G were used in the experiment and they were so chosen because of the wide range in their grades. The grades were identified in terms of the American Petroleum Institute (API) gravity, which is based on specific gravity values of the 250 to $275{ }^{\circ} \mathrm{C}(1 \mathrm{~atm})$ and the 275 to $300{ }^{\circ} \mathrm{C}(40 \mathrm{~mm})$ distillation fractions. The values of the API gravity of these crude oils were A: 49.32, B: 40.2, C: 40.0, D: 32.2, E: 29.4, F: 27.8, and G: 27.1, which corresponded to grades ranging from Super Light for A (the highest API gravity) down to Heavy for F and G (the lowest API gravity).

The thin capillary tubes made it possible for the interference patterns from the relatively opaque crude oils to be visible in transmission, while the design of the capillary-tube holder made it possible to record the interference patterns immediately after securing the crude oil sample in place, which negated any possibility of altering the temperature of the sample or volatizing its lighter aromatic compounds. The measurements on the oil samples were carried out independently from each other. Therefore the analysis of the resulting interference image of each crude oil sample was made on all the interference fringes to search for the correct interference order. Consulting the refractive index values obtained using an Abbe Refractometer for two of the crude oils, D and E did this. All samples were treated under the same exact experimental conditions of temperature $\left(20^{\circ} \mathrm{C}\right)$ and duration of exposure.

\section{RESULTS AND DISCUSSION}

Images of the resulting transverse interference patterns for all of the seven crude oils are shown in Fig. 2. The patterns comprises of only a few number of interference fringes, which were found to be typical for homogeneous liquids and could be explained in terms of twobeam type interference between pairs of laser rays passing through different optical media. Figure 3 shows the ray-tracing diagrams of a few of the possible combinations that can produce interference fringes. A fringe can be formed as a result of interference between R3, which passes entirely through the wall of the capillary tube, and R6, which passes perpendicularly through the middle of the capillary tube. Another fringe can be formed by the interference between a laser ray that enters the oil medium at an angle, i.e. R5, and one of the non-refracted rays that passes through the air on top of the capillary tube, i.e., R2, and a different fringe can also be formed due to interference between R4, a ray that suffers total internal reflection at the glass/oil interface, and another ray from the group that passes on top of the capillary tube, i.e., R1. The interference patterns shown in Fig. 2 corresponds to the upper half of the capillary tube only; those corresponding to the bottom half are symmetrically inverted in shape and are not shown in the figure.

The reason that the interference fringes are bell-shaped has to do with two combined factors. The first is the extended monochromatic light source in the $\mathrm{x}$-axis, which is the symmetry axis 
Military Technical College

Kobry Elkobbah,

Cairo, Egypt

May 29-31,2012

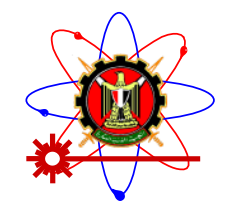

$6^{\text {th }}$ International Conference on Mathematics and Engineering Physics (ICMEP-6)

of the two capillary tubse, and the second is the curved surface area of the capillary tube, which makes the light rays impinge with different incident angles along the y-axis.

Here, we assume that the diffraction and scattering effects due to reflection are negligible. Consider a sheet of laser light is incident at point $F$ with incident angle $\theta_{F}$, where $-\arcsin \left(n_{g l} \frac{R_{i n}}{R_{\text {ou }}}\right)<\theta_{\mathrm{F}}<\arcsin \left(n_{g l} \frac{R_{\text {in }}}{R_{\text {ou }}}\right)$. At point $F$, the Cartesian coordinates $\left(X_{F}, Y_{F}\right)$ are then: $Y_{F}=R_{o u} \sin \theta_{F}$, and $X_{F}=-R_{o u} \cos \theta_{F}$, and Snell's law, applied at the air/glass interface, is:

$n_{a i} \sin \theta_{F}=n_{g l} \sin \varphi_{F}$,

where $\varphi_{F}$ is the reflection angle at point $\mathrm{F}$. Accordingly, the equation of a straight line that joins points $F$ and $G$ would be:

$Y=m_{1} X+b_{1}$,

where $m_{1}=-\tan \psi_{F}, b_{1}=\left(Y_{F}-m_{1} X_{F}\right)$ and $\psi_{F}=\theta_{F}-\varphi_{F}$.

Therefore, the Cartesian coordinates of point $\mathrm{G}$ then become:

$X_{G}=\frac{-m_{1} b_{1} \pm \sqrt{R_{i n}^{2}+m_{1}^{2} R_{i n}^{2}-b_{1}^{2}}}{1+m_{1}^{2}}$

and

$Y_{G}=\sqrt{R_{i n}^{2}-X_{G}^{2}}$

In equation (9), only the negative sign is of interest since $X_{G}$ ends up to the left of the origin O. For simplicity, we will consider $\overline{F G} \approx \overline{H I} \approx\left(R_{o u}-R_{i n}\right)$, which is the thickness of the capillary tube, and

$$
\overline{G H}=\sqrt{\left(X_{G}-X_{H}\right)^{2}+\left(Y_{G}-Y_{H}\right)^{2}} \approx 2|\overline{G Z}|,
$$

where $\mathrm{Z}$ is the intercept point with the $\mathrm{y}$-axis. Therefore, equation (5) reduces to:

$O P L_{5}=2 R_{\text {ou }}\left(1-\cos \theta_{F}\right)+2 n_{g l} \overline{F G}+2 n_{l i}\left|X_{G}\right|$.

Following the same reasoning, the optical path length of ray $\mathrm{R} 3\left(O P L_{3}\right)$ can be evaluated by expressing the Cartesian coordinates at point $\mathrm{B}\left(X_{B}, Y_{B}\right)$ as:

$X_{B}=\frac{-m_{2} b_{2}+\sqrt{R_{o u}^{2}+m_{2}^{2} R_{o u}^{2}-b_{2}^{2}}}{1+m_{2}^{2}}$,

$Y_{B}=\sqrt{R_{o u}^{2}-X_{B}^{2}}$,

where $m_{2}=-\tan \psi_{A}, b_{2}=\left(Y_{A}-m_{2} X_{A}\right)$, and $\psi_{A}=\theta_{A}-\varphi_{A}$, where this case in the range $\{-$ $\pi / 2, \quad-\pi / 2\}, \quad \theta_{\mathrm{A}} \quad$ will take the values $-\frac{\pi}{2}<\theta_{\mathrm{A}}<-\arcsin \left(n_{g l} \frac{R_{\text {in }}}{R_{\text {ou }}}\right) \quad$ and $\arcsin \left(n_{g l} \frac{R_{\text {in }}}{R_{\text {ou }}}\right)<\theta_{\mathrm{A}}<\frac{\pi}{2}$ 
Military Technical College

Kobry Elkobbah,

Cairo, Egypt

May 29-31,2012

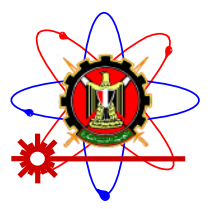

$6^{\text {th }}$ International Conference

on Mathematics and

Engineering Physics

(ICMEP-6)

Before an accurate analysis can be made the images in Fig. 2 had to be digitally processed. This was done by using MATLAB-based software that was developed to conduct a search for the maximum and minimum brightness of the fringes and to report them as functions of position ( $\mathrm{x}$ and $\mathrm{y}$ ). This allowed highly contrasted constructive and destructive interference fringes to be exactly defined. Figure 5 shows the outcome of this digital processing on the transverse interference patterns of crude oils D and F.

The absolute value of the refractive index of the medium enclosed within the capillary tube can be determined by measuring the deflection angles $(\varepsilon)$, defined as the angles between the R6 ray and the heights of the observed constructive (or destructive) interference fringes ( $y-z$ plane). To measure these deflection angles, in practice, it is convenient to employ Abel's transform as follows. For a light rays defined as orthogonal projection from geometrical wave fronts, the equation relating the path of these rays to the local index of refraction $(n)$ is ${ }^{18}$

$$
\frac{d}{d s}\left(n \frac{d r(s)}{d s}\right)=\nabla n
$$

where $r(s)$ is the position vector of a point on the ray and $s$ is the arc length of the ray. Choosing Cartesian coordinates system such that the axis of the capillary tube is in the $\mathrm{x}$ direction and the incident plane monochromatic wave is along the positive $\mathrm{z}$-axis, and using the paraxial approximation, $d s \approx d z$ this equation reduces to:

$$
\frac{d}{d z}\left(n \frac{d r}{d z}\right)=\nabla n
$$

where $r=x \mathbf{i}+y \mathbf{j}+z \mathbf{k}$, and $\nabla n=\frac{\partial n}{\partial x} \mathbf{i}+\frac{\partial n}{\partial y} \mathbf{j}+\frac{\partial n}{\partial z} \mathbf{k}$.

Since the impinging laser light into the capillary tube suffers refraction due to variation of refractive index in the z-direction, then at a particular height of the capillary tube $n$ can be taken as independent of $\mathrm{x}$. Then equation (17) becomes:

$$
\frac{d}{d z}\left(n \frac{d y}{d z}\right)=\frac{\partial n}{\partial y},
$$

Integration of this equation with respect to $z$ gives

$$
\frac{d y}{d z}=\frac{1}{n_{O}} \int \frac{\partial n}{\partial y} d x,
$$

In two-dimensional cylindrical coordinates, we have $r^{2}=z^{2}+y^{2}$. Consequently, $d z=\frac{r d r}{\sqrt{r^{2}-y^{2}}}$, and $\frac{\partial r}{\partial y}=\frac{y}{r}$.

If $\varepsilon$ is the angle of refraction of the rays that pass through the capillary tube, then

$$
\begin{aligned}
\varepsilon & =\frac{d y}{d z}=\frac{1}{n_{O}} \int_{-\infty}^{+\infty} \frac{\partial n}{\partial r} \frac{\partial r}{\partial y} d z=\frac{1}{n_{o}} \int_{-\infty}^{+\infty} \frac{\partial n}{\partial r} \frac{y}{r} \frac{r}{\sqrt{r^{2}-y^{2}}} d r \\
& =\frac{2 y}{n_{O}} \int_{0}^{+\infty} \frac{\partial n}{\partial r} \frac{d r}{\sqrt{r^{2}-y^{2}}} .
\end{aligned}
$$

This is the form of Abel's transform whose inverse is ${ }^{19}$ 


\section{Military Technical College \\ Kobry Elkobbah, Cairo, Egypt \\ May 29-31,2012}

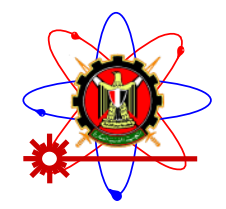

$6^{\text {th }}$ International Conference on Mathematics and Engineering Physics (ICMEP-6)

$$
\frac{n(r)-n_{f}}{n_{f}}=-\frac{1}{\pi} \int_{i}^{f} \varepsilon \frac{d y}{\sqrt{y^{2}-r^{2}}},
$$

where $i=r$ and $f=\infty$ are the limits of the phase object and $n_{f}$ is the index of refraction at $f$, which is $n_{\mathrm{gl}}=3 / 2$ in our case

By using Eq. (21) the refractive indices of the seven crude oils were determined as $\mathrm{A}(1.446870), \mathrm{B}(1.453822), \mathrm{C}(1.460520), \mathrm{D}(1.474833), \mathrm{E}(1.481889), \mathrm{F}(1.488885)$, and G(1.498860). Figure 6 shows a plot of the refractive index as a function of the crude oils' API gravity, where the line represents, arbitrarily, a polynomial fit of second order. The plot demonstrates a decrease in refractive index with the increase of API gravity as expected. The accuracy of these data points is based on the parameters that define the locations of the fringes i.e., $\varepsilon$, y and $r$, which in turn correspond to displacements that are proportional to $1 / 2 \lambda$, and is estimated to be $5 \times 10^{-6}$. It was important that one of the interference patterns be recorded with an actual length scale displayed in the background so that the magnification factor of the recorded images could be determined. Another important point to mention here is the accuracy of the digitally reported values of the brightness in the images. Care should be taken not to saturate the CCD camera with intense light, as this would compromise the validity of the reported brightness values.

Finally, it is interesting to note that, under the same experimental conditions, the number of interference fringes appearing in the images of the crude oils is found to be considerably fewer than those appearing in the case of optical fibers ${ }^{12}$. This is due to the fact that in optical fibers the refractive index changes gradually with the radius of the fiber core, while in the crude oil this change occurs in more discrete steps. In other words, if the inside of the fiber were to be made out of crude oil then one would be able to describe the change in the refractive index simply in terms of discrete layers each of which has a constant refractive index. We estimate the widths of these layers to be $\sim 0.5 \mu \mathrm{m}$ for the samples used in this work.

\section{CONCLUSION}

The work in this paper represents the first attempt to measure the refractive index of the relatively opaque crude oil using a capillary tube interferometer. The oil-filled capillary tubes were illuminated by a thick HeNe laser sheet and the resulting transverse interference patterns were projected on a screen whose plane was perpendicular to that of the laser sheet. As expected, the patterns showed bell-shaped fringes as in the case of optical fibers except that the number of the fringes in this case was much smaller and they did not show dips at the center. The characteristic bell shape was demonstrated mathematically by calculating the optical path difference between a reference ray and a ray that passes through the oil sample. The refractive indices of the crude oil samples were determined with accuracy of $5 \times 10^{-6}$ by measuring the deflection angles of the fringes and then by using Eq. 21. The high accuracy obtained makes this technique a valuable one for measuring the refractive index of crude oil without having to extrapolate it from the oil's thermal distillates. It may also prove useful as a tool for fingerprinting crude oils. 

Military Technical College
Kobry Elkobbah,
Cairo, Egypt
May 29-31,2012

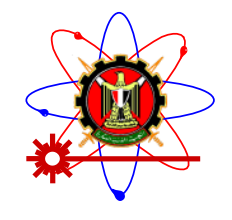

$6^{\text {th }}$ International Conference on Mathematics and Engineering Physics (ICMEP-6)

\section{ACKNOWLEDGEMENTS}

The authors gratefully acknowledge KFUPM's support in carrying out this research. E.H. wishes also to acknowledge the support of the Research Institute of King Fahd University of Petroleum and Minerals.

\section{REFERENCES}

1) Speight JG, "The Chemistry and Technology of Petroleum", New York, M. Dekker, 1980.

2) Otremba Z, Optics Express, 2000; 7: 129 - 134.

3) Buckley JS, Energy and Fuels, 1999; 13: 328 - 332.

4) Buckley JS, Hirasaki GJ, Liu Y, Von Drasek S, Wang JX, and Gill BS, Petroleum Science and Technology, 1998; 16: 251- 285.

5) Deng Y, Li B, Appl. Opt., 1998; 37(6): 998 - 1005.

6) Tarigan H, Neill P, Kenmore CK, Bornhop DJ, Anal. Chem, 1996; 68: 1762-1770.

7) Menn ML, and Lotrian J, J. Phys. D: Appl. Phys. 2001; 34: 1256-1265.

8) N. Barakat and A. A. Hamza: Interferometry of Fibrous Materials (Bristol: Adam Hilger, 1990).

9) Barakat N, El-Ghandoor H, Hamed AM, and Labib S, Exp. in Fluids, 1993; 16: 42 45.

10) Alexandrov SA, and Chernyh IV, Opt. Eng., 2000; 39: 2480 - 2486.

11) Zaiat SY, and El-Henawi HA, Meas. Sci. Technol., 1996; 7(8): 1119-1123.

12) El-Ghandoor H, El-Ghafar EA, and Hassan R, Opt. Laser Technol, 1999; 31: 481 488.

13) El-Ghandoor H, Nasser I, Rahman MA, and Hassan R, Opt. Laser Technol, 2000; 32: $281-286$.

14) Born M, and Wolf E, "Principle of optics," New York: Pergamon 1970 [Chapter 3, p. 122 and chapter 2, p. 87]

15) Bracewell R, "the Fourier and its applications," New York: McGraw-Hill, 1978. p.262

\section{Figure Captions}

Figure 1: Schematic diagram of the experimental setup.

Figure 2: Raw images of the transverse interference patterns for all the seven crude oils as recorded using the CCD camera.

Figure 3: Ray tracing of selected rays impinging on the upper hemisphere of the capillary tube. The locations of three possible fringes are shown on the screen at the right.

Figure 4: Optical path differences $O P L_{5}-O P L_{1}$ and $O P L_{3}-O P L_{1}$ plotted as functions of incident angle for the values $\mathrm{R}=\mathrm{R}_{\mathrm{ou}} / \mathrm{R}_{\mathrm{in}}=1.8, n_{\mathrm{gl}}=1.5$ and $n_{\mathrm{li}}=1.477$. The curved pattern between the two middle solid lines simulates the bell-shaped interference fringe, which is due to interference between a ray passing through the glass and oil, i.e., R5, and a reference ray, e.g., R1. The tails outside the solid lines simulates an interference fringe of different shape resulting from the interference between a ray passing through the glass wall of the capillary tube, i.e. R3, and R1. Figure 4: Examples of highly contrasted interference patterns (crude oils D and F) 
Military Technical College Kobry Elkobbah,

Cairo, Egypt

May 29-31,2012

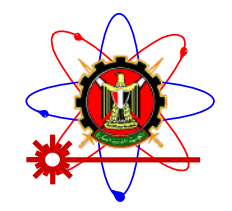

$6^{\text {th }}$ International Conference on Mathematics and Engineering Physics (ICMEP-6)

produced by digitally processing the raw images.

Figure 5: A Plot of the oil's refractive index as a function of its API gravity 